\title{
Preliminary Design of the Beam Loss Monitoring System for the SNS ${ }^{\dagger}$
}

\author{
R. WITKOVER*, D. GASSNER \\ BNL, Upton, $N Y$, *TechSource Inc., Santa Fe, NM
}

\begin{abstract}
The SNS to be built at Oak Ridge National Laboratory will provide a high average intensity $1 \mathrm{GeV}$ beam to produce spallation neutrons. Loss of a even small percentage of this intense beam would result in high radiation. The Beam Loss Monitor (BLM) system must detect such small, long term losses yet be capable of measuring infrequent short high losses. The large dynamic range presents special problems for the system design. Ion chambers will be used as the detectors. A detector originally designed for the FNAL Tevatron, was considered but concerns about ion collection times and low collection efficiency at high loss rates favor a new design. The requirements and design concepts of the proposed approach will be presented. Discussion of the design and testing of the ion chambers and the analog front end electronics will be presented. The overall system design will be described.
\end{abstract}

\section{BACKGROUND}

The Spallation Neutron Source (SNS) ${ }^{1}$ is an accelerator based intense neutron source being built at Oak Ridge National Laboratory (ORNL) by a collaboration of 6 national laboratories. The controls and some beam instrumentation were consolidated so that the same detectors and interface hardware would be used throughout the facility to minimize costs and reduce uncertainty due to different instrumentation. BNL has responsibility for the beam loss monitoring system for all of SNS. The SNS $\mathrm{H}$-minus ion source will inject a $60 \mathrm{~Hz}$ chopped beam into a $2.5 \mathrm{MeV}$ RFQ. After acceleration to $87 \mathrm{MeV}$ in a Drift Tube Linac (DTL), the beam enters the Cavity Coupled Linac (CCL), leaving at $186 \mathrm{MeV}$. The Superconducting RF Linac (SRF) will increase the beam energy to $1 \mathrm{GeV}$. The beam is transported to the Ring via HEBT, converted to protons using a stripping foil, and accumulated in a single 695 nsec bunch over the $1 \mathrm{msec}$ injection pulse. The bunch is transported via the RTBT line to the spallation neutron target. The design peak beam current in the Linac and HEBT will be $38 \mathrm{~mA}$. In the Ring it will increase to more than 40 A (average) at the end of the pulse, for a total of $1.5 \times 10^{14}$ protons in the single bunch. The BLM system will be designed for an intensity of $2 \times 10^{14}$ protons for future expansion.

The baseline design will produce $1.4 \mathrm{MW}$ at a $60 \mathrm{~Hz}$ rate. This very high average beam power makes it crucial that uncontrolled losses do not produce high activation, preventing hands-on maintenance. This will be done by minimizing losses through

\footnotetext{
${ }^{4}$ Work performed under the auspices of the U. S. Dept. of Energy
} 
careful design and providing beam dumps and collimators where controlled losses can be contained. The BLM system must minimize Un-Controlled losses by providing data for tuning the machine and inhibiting the beam when excessive losses occur.

\section{SYSTEM PHILOSOPHY AND DESIGN}

The BLMs will be the primary tool for limiting uncontrolled losses if there is sufficient detector coverage in all sections of the SNS. To achieve this, the AGS Linac and Ring use extended ion chambers made from hollow argon-filled, large diameter coaxial cables ${ }^{2}$. ISIS, a machine similar in function to SNS, has used the same type detectors $^{3}$. A "line" detector has $1 / \mathrm{r}$ spatial dependence but their length often close coupling to the beam line, lowering their response. Since most losses are at the quad locations, most of the detector length receives a much lower exposure. The connectors for the coax cable BLM cannot support high bias voltage, limiting the linear range to lower dose rates. For these reasons smaller, sealed glass ion chambers designed for the FNAL Tevatron were used in $\mathrm{RHIC}^{4}$, but more detectors were required to obtain similar coverage. In the SNS Linac, HEBT, Ring and RTBT, as in RHIC, detectors will be placed at essentially every quadrupole (beta-max) and at other key points. Provision will be made for a number of movable BLMs which can used for more detailed study.

Ion chambers were chosen as the detectors over solid state (pin diode) and scintillator-photomultipliers. A commercial pin-diode $\mathrm{BLM}^{5}$ is available but the 10 $\mathrm{MHz}$ maximum output rate would not provide the dynamic range required during a 1 msec beam pulse. Scintillator-photomultipliers do not the long term stability needed for an accelerator application. However, to be able to see losses within the bunch, scintillator-photomultipliers will be installed at a limited number of points, such as, Ring injection and extraction, and other strategic points. Since only a relative time history is of interest for this application, non-calibrated data is acceptable.

\section{System Requirements}

High level losses over several pulses can damage or quench the super-conducting cavities in the SRF Linac. Low level losses can produce excessive activation and prevent "hands-on" maintenance. To prevent this, losses must be limited to $1 \mathrm{~W} / \mathrm{m}$ averaged over several seconds duration. This is roughly equivalent to $10^{-4}$ of $2 \times 10^{14}$ protons per pulse lost uniformly around the Ring. Sensitivity must be available to measure such long-term, low level losses yet not saturate under short duration, high level losses. These considerations lead to the requirements shown below.

Detailed time history. Data within a macro-pulse will be acquired at 100 $\mathrm{kSa} / \mathrm{S}$ per channel but it will be packed and transmitted at a $6 \mathrm{~Hz}$ rate.

Total loss for each BLM per macro-pulse. Each BLM's data will be summed at the front end computer (IOC) and transmitted once per second. This data 
will be presented as a "waterfall" display ${ }^{6}$ showing detector location on the horizontal axis, Time on the vertical axis, and loss intensity as color. A display of detector location on the $\mathrm{x}$-axis and loss on the $\mathrm{y}$-axis may also be required for update at once or more per second. A 1000 point FIFO history of the losses for each BLM will be kept at the console level for use inthe event of an abort.

Long-term, low-level beam loss alarm and display. The long-term, lowlevel beam loss $(<1 \mathrm{~W} / \mathrm{m})$ will be using a 24-bit ADC. This data will be averaged over 10 seconds in the IOC and compared against a $1 \mathrm{~W} / \mathrm{m}$ reference. Warnings will be sent as this level is approached and an alarm when it is exceeded. The pre-averaged low-level data will be available as a water-fall display or a strip chart style display.

Fast Loss Output for the Machine Protect System. Each BLM electronics channel will provide an output signal to the Machine Protect System (MPS) to shut off the beam in the event of a fast high-level beam loss. This signal will be derived from the integrated beam loss and shall be occur within 5-10 $\mu$ sec of the occurrence. Each channel shall have be capable of being masked to allow the use of diagnostic hardware which cause beam loss, such as wire scanners,. The mask will also permit studies which might generate a higher than normal local beam loss, and to disable malfunctioning BLM channels which would prohibit beam operation. Masking will be done within the MPS hardware.

Gain setting and readback for each BLM. Each BLM electronics channel will have the capability of 3 jumper selected gains to compensate for high radiation locations, such as at dumps, injection, extraction, and the collimators, or lower radiation locations such as lower energy regions and well shielded areas. The "Viewing Gain" can be changed (x1, x10) via the control system without affecting the fast loss trip or the $1 \mathrm{~W} / \mathrm{m}$ level sensing. Gain setting may be required pulse-to-pulse to allow for different mode cycles but is not required during the macro-pulse. Read back of the jumper-selectable gain and Viewing Gain states will be provided.

Bias Voltage Control. During studies periods, it may be necessary at times to vary the $\mathrm{HV}$ bias Voltage. Readback of the voltage, current and polarity will also be needed. Each rack location will contain at least 2 HV Bias Supplies, powering alternate BLM detectors. The BLM System can be tested by switching the HV Bias Power Supplies Off and On again. The resulting transient capacitively couples between the high voltage and signal electrode and through the BLM electronics, checking all components of the system, including the bias and signal cables.

System Calibration. Each BLM detector will be calibrated in a laboratory test setup using a static radiation source. Data will be taken across the operating voltage range for each detector. Initial testing will be done at BNL until the production is stable with subsequent units tested at ORNL. Insitu tests of the 
system through the installed cabling will be performed using a calibrated $1 \mathrm{G}$ Ohm resistor and voltage source for use in the calibration database.

\section{Expected Losses}

Experience at LAMPF and the PSR, and studies for APT at $\mathrm{LANL}^{7}$ have determined that losses of $1 \mathrm{~W} / \mathrm{m}$ will allow hands-on accelerator maintenance $(\sim 100$ $\mathrm{mR} / \mathrm{hr}$ at $1 \mathrm{foot}$ ), with transverse losses primarily at quadrupoles where the beta-max occurs $^{8}$. The beam-on dose rate can be estimated from the beam-off dose rate by a "rule-of-thumb": multipliers of 500-1000 are typically used. Taking $100 \mathrm{mR} / \mathrm{hr}$ at 1 foot as the beam-off activation, the beam-on dose rate may be estimated at $100 \mathrm{R} / \mathrm{hr}$, or $0.46 \mathrm{mR} /$ pulse, which is equivalent to $0.46 \mathrm{R} / \mathrm{sec}$ during the $1 \mathrm{msec}$ pulse.

The upper end loss has been specified as $1 \%$ local loss based on the expected losses in the Ring collimators ${ }^{9}$. The BLM system must generate a beam abort signal for the MPS in less than $10 \mu \mathrm{sec}$. This will be done by integrating the beam loss during the macropulse and comparing the result to a programmable reference. Longer term losses at lower level can also do damage to beamline components. The numerically integrated output of each BLM will be compared to settable thresholds in the local processor. Excessive loss will generate an abort signal to the MPS through a VME utility module in the front end processor crate. Still lower level losses, corresponding to the $100 \mathrm{mR} / \mathrm{hr}, 1 \mathrm{~W} / \mathrm{m}$ loss will be similarly monitored and a softeware warning provided to the operators.

\section{Detector}

SNS will usc 255 (Linac 88, HEBT 52, Ring 75, RTBT 40) argon-filled ion chambers as the primary detectors for monitoring beam losses. Argon has the advantage of fast electron transit time compared to slower air filled detectors ${ }^{10}$. The initial choice was an ion chamber designed by Shafer ${ }^{11}$ at FNAL in 1982 for the Tevatron, and modified to improve radiation hardness and reduce noise for RHIC at $\mathrm{BNL}^{12}$. However there was concern about saturation at high dose rate and the ion transit time $(\sim 700 \mu \mathrm{sec}$ at $2 \mathrm{kV}$ bias $)$. The ions, constituting $25 \%$ of the signal, would appear as a continued rise during the $1 \mathrm{msec}$ pulse followed by a long ion tail after. However, processing could unfold the pulse shape. A new ion chamber, designed to overcome these limitations ${ }^{13}$, utilizes a larger inner diameter electrode to significantly decrease the ion transit time and raise the collection efficiency for a $1 \%$ local loss.

Figure 2 shows the measured response the $200 \mathrm{MeV}$ BNL Linac to Booster line for the FNAL and a new prototype detector with nitrogen fill at the equivalent if a $0.05 \%$ local loss. The slow rise during and long tail after the beam pulse is clearly seen for the FNAL detector. The "noise" is chopper modulation of the beam. 

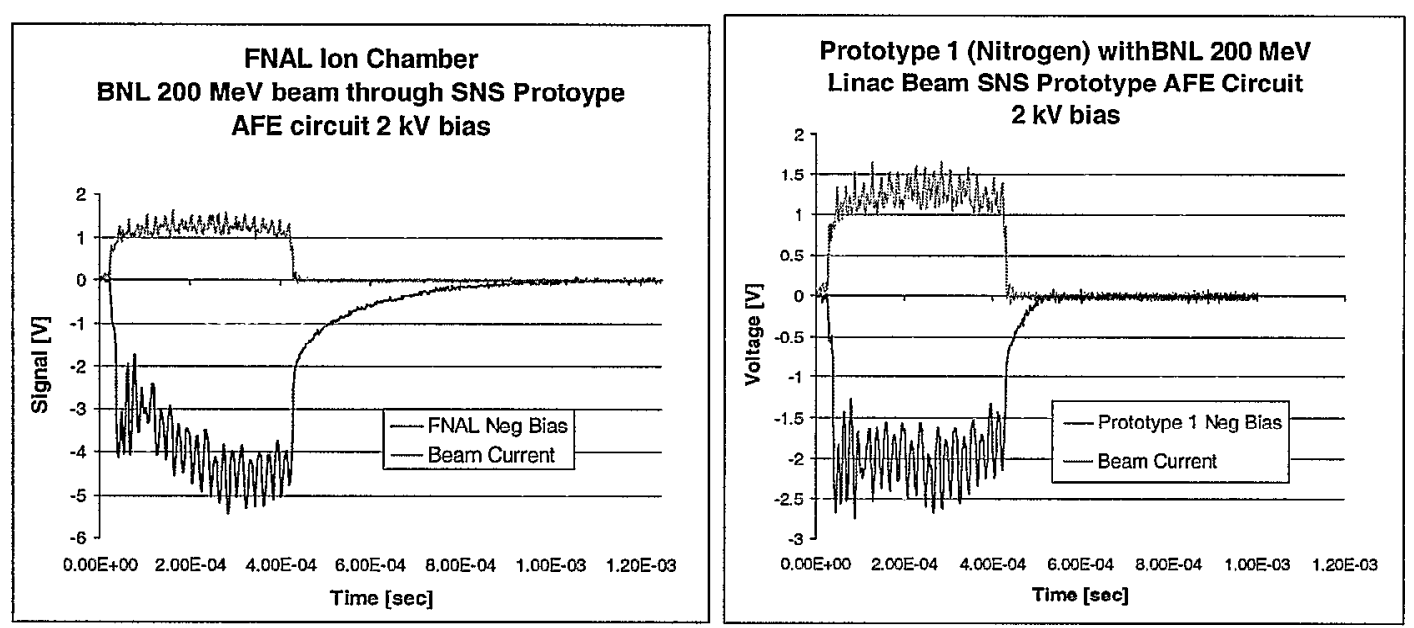

FIGURE 2. Beam Tests of the FNAL and New Prototype Ion Chambers

\section{Installation}

The detectors may be mounted directly to the beam pipe, magnet, or moved away from the radiation source to reduce signal output, allow a wider view over a larger area without intervening shielding. A typical SNS installation might raise the BLM 30 $\mathrm{cm}$ above the beam line on the upstream side of a quad. BLMs will be placed so that successive units can have overlapping views to allow limited coverage in the event a channel fails. The system will be packaged so that adjacent detectors will not be placed on consecutive electronics channels in the same circuit module, where possible.

Some BLMs may be exposed to X-rays from RF cavities. Measurements at BNL indicate that this may reach $50 \mathrm{R} / \mathrm{hr}$ during beam time. A study is needed to determine the type and amount of shielding that would reduce the X-ray contribution to an acceptable level, and its effect on the beam loss flux.

\section{Cabling}

Low tribo-electric RG59 type cable such as Belden 9054 or 9224 will be used for the signals. Tribo-electric noise comes from friction between the conductors and insulation due to movement, such as vibration, but a low friction coating between insulator and conductors will reduce this significantly. Two HV cables (RG-59), from separate HV power supplies will connect to alternate BLM's from each rack to provide some coverage in the event of a high voltage short or power supply failure.

\section{ELECTRONICS}

The BLM system will be distributed throughout the SNS. The controls electronics will be in VME, with analog signal conditioning housed in a non-VME crate in the same rack. The analog crate will use linear rather than switching power supplies because of the very small signal currents. Figure 3 shows the system block diagram. 


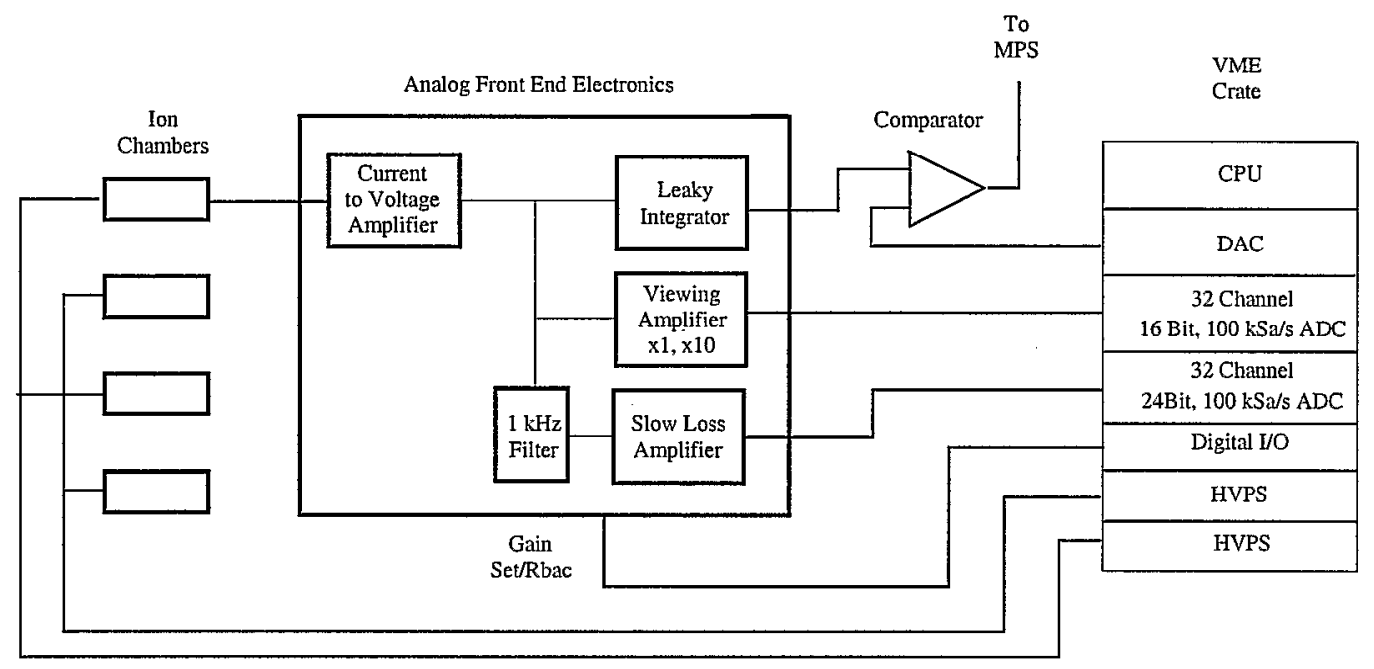

Figure 3. BLM System Block Diagram

\section{System Dynamic Range Requirements}

The BLM System must measure beam losses from a $1 \%$ local loss down to $1 \%$ resolution of a $1 \mathrm{~W} / \mathrm{m}$ loss, a dynamic range of $2 \times 10^{6}$, or at least 21 bits. The design exploits the difference in bandwidth between these limits to allow additional resolution at the low end. During the $1 \mathrm{msec}$ beam pulse the $1 \mathrm{~W} / \mathrm{m}$ dose rate is estimated as $0.46 \mathrm{R} / \mathrm{sec}$ at $1 \mathrm{ft}$. For a typical BLM sensitivity of $70 \mathrm{nA} / \mathrm{R} / \mathrm{sec}$, this corresponds to $32.4 \mathrm{nA}$ signal current during the pulse. The upper end loss, allowing for future improvements to $2 \mathrm{MW}$ beam power, corresponds to $20 \mathrm{~kW}$. Since the signal must inhibit the beam within $10 \mu \mathrm{sec}$, only the electron signal will be considered, reducing the BLM sensitivity to $35 \mathrm{nA} / \mathrm{R} / \mathrm{sec}$. Then $\mathrm{I}_{\mathrm{Max}}=16.2 \times 10^{-9} \times 2$ $\times 10^{4}=0.324 \mathrm{~mA}$ for a loss of $1 \%$ uniformly during the $1 \mathrm{msec}$ pulse.

\section{Analog Front End Electronics}

Figure 4 shows the AFE electronics schematic. The circuit provides 3 outputs to meet the fast trip, wideband-wide range data and $1 \mathrm{~W} / \mathrm{m}$ sensing requirements.

\section{Wideband Signal Circuit}

Since the ion chamber is a current source, the input resistor doesn't affect the signal gain but does determine the voltage noise gain and input signal risetime. A $100 \mathrm{~m}$ cable and $470 \Omega$ resistor gives about a $5 \mu \mathrm{sec}$ risetime. A $6.2 \mathrm{k} \Omega$ feedback resistor puts the signal mid-range for $5 \mathrm{~V} \mathrm{ADC}$ for a $1 \%$ beam loss. For the $50 \mathrm{kHz}$ SNS BW, the $10 \mathrm{pA}$ at $10 \mathrm{~Hz} \mathrm{BW}$ noise observed in RHIC would correspond to $4.36 \mu \mathrm{V}$. Three jumper selectable gains with readbacks are provided. "Viewing Gain" can be set and read back remotely without affecting the beam interrupt or $1 \mathrm{~W} / \mathrm{m}$ outputs. The signals of Figure 2 were made using this circuit. 


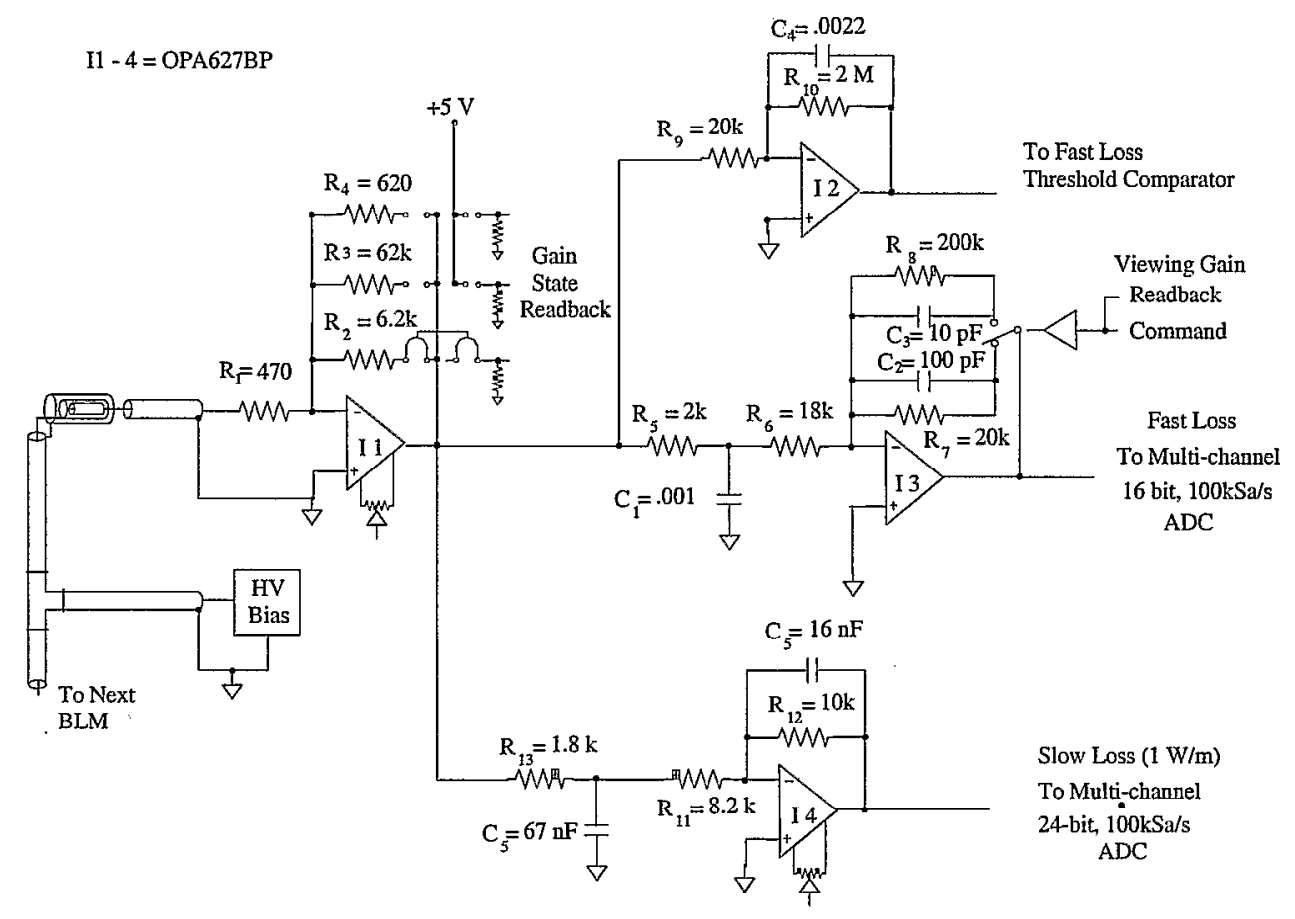

Figure 4. Analog Front End Electronics Schematic

\section{The $1 \mathrm{~W} / \mathrm{m}$ loss Circuit}

A $1 \mathrm{~W} / \mathrm{m}$ loss will produce $200 \mu \mathrm{V}$ out of the input stage, roughly an LSB for a $5 \mathrm{~V}$, 16-bit (15-bit plus sign). Another 7-bits would be needed for $1 \%$ resolution of a 1 $\mathrm{W} / \mathrm{m}$ loss. Using a $1 \mathrm{kHz}$ low pass filter, the noise would be reduced by the equivalent of almost 3-bits. Having an analog filter precede the output amplifier reduces the peak of short duration losses, easing $\mathrm{ADC}$ saturation. A lower cut-off might not allow sufficient time to measure the baseline for offset subtraction. The output goes to a 24 bit, $100 \mathrm{kSa} / \mathrm{sec} \mathrm{ADC}$. Tests of one ADC (ICS-110B) indicate it can achieve 18-19 bit resolution. By sampling over 10 seconds or longer, the required resolution could be obtained. Detailed testing of this and other ADCs, including thermal drift of offset and gain, is in process. Using measurements prior to the pulse, offsets of the AFE as well as the ADC can be measured and subtracted. Dedication of one ADC channel to read a reference voltage may be required to compensate for gain thermal drift.

\section{The Pulse Integrated Dose Circuit}

Experience at $\mathrm{LANSCE}^{14}$ has shown that a beam inhibit signal should be based on integrated dose rather than dose rate. Thus an integrator will be used to provide a signal to a comparator to generate a signal for the MPS when the programmable reference is exceeded. (See Figure 3.) A gated integrator with a triggered reset would give a precise integral of the loss, but the gating, reset, and charge injection compensation circuitry add considerable complexity to the design. A simple "leaky 
integrator", using a large value resistor to bleed the charge, eliminates this and provides an adequate representation of the pulse dose. Simulations indicate that for an $\mathrm{RC}$ time constant of $1 / 3$ the pulse period, the output will reach equilibrium in 3 beam pulses with an error of less than $10 \%$ while decaying to under $5 \%$ by the next beam pulse. This is a reasonable trade-off to the reduction of complexity and reliability of the triggered integrator.

\section{ACKNOWLEDGMENTS}

The authors would like to $R$. Shafer and M. Plum of LANL for providing valuable insight and discussions regarding the topics in this work. Yongbin Leng (BNL) evaluated the ADCs for this project. John Smith of BNL has been invaluable in supporting the Controls aspect of this system.

\section{REFERENCES}

\footnotetext{
${ }^{1}$ Alonso, J., "The Spallation Neutron Source Project", Proceedings of the 1999 PAC, NY, 1999, p574. For current status see http://www.sns.gov

${ }^{2}$ Witkover. R. L. ,"Microprocessor Based Beam Loss Monitor System for the AGS", IEEE Trans. Nucl. Sci 263313 (1979)

${ }^{3}$. M. A. Clarke-Gayther, et al. "Global Beam loss Monitoring Using Long Ionisation Chambers at ISIS", $4^{\text {th }}$ European Particle Accelerator Conference, London, England, 1994, p1634

${ }^{4}$ Witkover, R. L., Michnoff, R. J. and Geller, J. M., "RHIC Beam Loss Monitor System Initial Operation", Proceedings of the 1999 PAC, NY, 1999, 2247

${ }^{5}$ Bergoz Inc, http://www.bergoz.com

' Bai, M., et al., "RHIC Beam Loss Monitor System Commissioning in the Year 2000 Run", To be published these proceedings.

${ }^{7}$ Hardekopf, R. A., "Beam Loss and Activation at LANSCE and SNS", LA-UR-99-6825, Los Alamos National Laboratory, Los Alamos, NM. Sept. 1999

${ }^{8}$ Loc. Cit. Reference 7

${ }^{9}$ Y. Y. Lee, BNL, Private Communication, Oct 2001

${ }^{10}$ Plum, M., and Brown, D., "Response of Air-Filled Ion Chambers to High Intensity Radiation Pulses", Proceeding of the 1993 PAC, p2181

11 . Shafer, R. E., et al., "The Tevatron Beam Position and Beam Loss Monitoring Systems", Proc. The $12^{\text {th }}$ Int'l Conf. High Energy Accel., p609, 1983

${ }^{12}$ Loc. Cit. reference 4.

${ }^{13}$ Witkover, R. L. and Gassner, D. "Design of an Improved lon Chamber for the SNS", These proceedings

${ }^{14}$ Plum, M., and Shafer, R., Private communications.
} 